\title{
O Cultivo do Mogno Africano (Khaya spp.) e o Crescimento da Atividade no Brasil
}

\author{
Andressa Ribeiro ${ }^{1}$, Antonio Carlos Ferraz Filho $^{2}$, José Roberto Soares Scolforo ${ }^{2}$ \\ ${ }^{1}$ Departamento de Engenharias - CPCE, Universidade Federal do Piauí - UFPI, Bom Jesus/PI, Brasil \\ ${ }^{2}$ Universidade Federal de Lavras - UFLA, Lavras/MG, Brasil
}

\begin{abstract}
RESUMO
O setor florestal brasileiro está em plena expansão e com um aumento gradativo de investidores florestais optando pelo cultivo de espécies de madeira nobre. O mogno africano (Khaya spp.) é uma espécie que vêm se destacando na preferência dos empresários como opção no investimento florestal. Porém, estudos e pesquisas sobre a espécie, principalmente no Brasil, são escassos. Assim, a presente revisão procurou reunir diversas fontes de publicação, nacionais e internacionais, abordando aspectos históricos do mogno, buscando aclarar as características da espécie e a experiência de outros países no manejo dessa cultura.
\end{abstract}

Palavras-chave: mogno africano (Khaya spp.), plantios florestais, manejo florestal.

\section{African Mahogany (Khaya spp.) Cultivation and the Increase of the Activity in Brazil}

\begin{abstract}
The Brazilian forest sector is fast growing with a gradual increase of forest investors choosing valuable hardwood species for tree crops. African mahogany (Khaya spp.) is a species that has been preferred by many entrepreneurs as a forestry investment. However, there are few studies and research on the species, especially in Brazil. Therefore, this review aimed to bring together diverse sources of national and international publications, discussing the history of the mahogany and characteristics of the species as well as the experience of other countries on the management of this crop.
\end{abstract}

Keywords: african mahogany (Khaya spp.), forest plantations, forest management. 


\section{INTRODUÇÃO}

O mogno africano (Khaya spp.), do qual uma das espécies é a Khaya ivorensis A. Chev., éárvore de origem africana pertencente à família botânica Meliaceae, mesma família do mogno nativo, da andiroba e do cedro. Possui madeira nobre de grande potencial econômico para comercialização interna e externa, podendo ser empregada na indústria moveleira, naval, construção civil, painéis e laminados, entre outros usos (Pinheiro et al., 2011).

No Brasil, a espécie teve seus primeiros plantios instalados na região Norte no ano de 1976 e a crescente demanda por madeira tropical está levando a novos investimentos em plantios comerciais de mogno africano em todo o país, aquecendo o mercado florestal em torno da espécie. Diversos plantios de Khaya spp. já foram instalados na Austrália, Ásia e América tropical, porém poucos estudos foram publicados discutindo resultados dos sistemas silviculturais adotados, do crescimento e da produtividade, da análise econômica e demais que forneçam aos manejadores e investidores florestais mais informações para a tomada de decisão na condução da espécie.

Dessa forma, a presente revisão é uma reunião de informações encontradas na literatura sobre a essência florestal, enfocando principalmente a espécie Khaya ivorensis, devido ser essa a mais difundida, até o presente, no Brasil.

\section{A ORIGEM DA NOMENCLATURA MOGNO}

Lamb $(1963,1967)$ se propôs o desafio de desvendar a verdadeira origem da nomenclatura mogno, e, após ampla discussão sobre a história da colonização da Jamaica pelos europeus, afirmou que a raiz linguística da palavra mogno provém da palavra ogan, empregada pelas tribos nigerianas Yorubas e Ibo. Tais tribos integravam uma porção considerável dos escravos africanos levados para a ilha jamaicana no período de colonização (século XVI), e esses utilizavam a palavra moganwo para designar a fartura de árvores e madeira de mogno presentes na floresta.

Em 1655, após a tomada da ilha da Jamaica pelos ingleses, o termo mogano foi adaptado para mahogany para designar esse importante elemento da vegetação local, detentor de excelente qualidade para fabricação naval e civil, a Swietenia spp. Naquele tempo, a distinção pelos escravos entre as árvores do gênero Khaya e Swietenia não existia, pois, uma vez fixados na Jamaica, encontraram características semelhantes entre as espécies, inclusive no uso medicinal dos extrativos contidos na casca (Lamb, 1963, 1967).

O processo exploratório do mogno (Swietenia spp.) foi crescente ao longo do século XVI. A expansão do comércio internacional e a crescente demanda por essa madeira de propriedades nobres resultou no esgotamento das fontes de fácil acesso ao longo dos rios, conduzindo assim, esforços para desenvolver fontes alternativas para abastecer de madeira o mercado consumidor (Lamb, 1963; Revels, 2003). Portanto, em meados de 1880, comerciantes de madeira da Inglaterra e demais países europeus começaram a buscar outras fontes madeireiras nas colônias africanas. Assim, o termo mahogany passou a designar, além do gênero Swetenia, as madeiras africanas do gênero Khaya (Lamb, 1963). Atualmente, a nomenclatura mogno é empregada apenas para madeiras e árvores de florestas tropicais americanas e africanas dos gêneros Khaya e Swietenia (Lamb, 1963; Ward et al., 2008).

A controvérsia com demais madeiras tropicais que utilizavam mogno como nome, tal como mogno das Filipinas, finalizou-se em 1963, garantindo ao comércio de madeira a padronização do produto designado como mogno (Lamb, 1963, 1967). A FAO (2001) reconheceu que a família Meliaceae pode ser chamada de "família dos mognos" e distinguiu os principais gêneros conforme a região (África e América Latina). Ward et al. (2008) afirmaram que as espécies de madeira tropical pertencentes à subfamília Swietenioideae (mognos verdadeiros) incluem algumas das melhores madeiras para movelaria no mundo, com base nas características geralmente compartilhadas, tais como estabilidade dimensional e trabalhabilidade. A subfamília inclui os gêneros Cedrela e Swietenia nos neotrópicos, Entandrophragma, Khaya e Lovoa na África e Chukrasia e Toona na Austrália. Reilly \& Robertson (2006) afirmaram que os mognos da América do Sul (Swietenia spp.) e África (Khaya spp.) são bastante conhecidos nos mercados globais de madeira e já são negociados por séculos. 


\section{CARACTERÍSTICAS DO GÊNERO KHAYA}

O gênero Khaya spp. é denominado por muitos autores mogno africano (Lamprecht, 1990; Falesi \& Baena, 1999; FAO, 2001; Opuni-Frimpong et al., 2008b; Pinheiro et al., 2011). Segundo Fremlin (2011), existem cinco espécies de mogno africano, denominadas: K. senegalensis, K. anthotheca, K. grandifoliola, K. ivorensis e K. madagascarensis. Porém, para alguns autores o gênero se divide em apenas quatro espécies (Pinheiro et al., 2011; Falesi \& Baena, 1999) ou mesmo em seis espécies (Wiselius, 1998 apud Khairul Alam et al., 2012), das quais quatro pertencem aos trópicos africanos e duas à União das Comores e Madagascar. Segundo a lista vermelha de espécies ameaçadas da IUCN (2013), a espécie K. madagascariensis é a única classificada como ameaçada, as demais espécies de Khaya são classificadas como vulneráveis à extinção apenas em seu local de origem.

Acajou D'Afrique (1979), Lamprecht (1990), Orwa et al. (2009) e Pinheiro et al. (2011) descreveram os aspectos ecológicos, silviculturais e tecnológicos das diferentes espécies do gênero Khaya de forma detalhada. O gênero pertence à família Meliaceae e têm origem em diferentes países africanos (Ibrahim et al., 2006). De maneira geral, as árvores atingem grandes dimensões, com altura variando de 30 a 35 metros, podendo chegar à altura superior a 60 metros, o tronco pode atingir 2 metros de diâmetro e, geralmente, possuem sapopemas na base, para garantir a sustentação. As folhas são parepinadas, sendo decíduas ou sempre verdes, dependendo da espécie. As flores são produzidas em inflorescências em panículas, o fruto é globoso e possui 5 a $8 \mathrm{~cm}$ de diâmetro, contendo 4 ou 5 valvas preenchidas com numerosas sementes aladas e achatadas (Lemmens, 2008; Pinheiro et al., 2011; CABI, 2013).

O mogno africano, aqui nos referindo a Khaya ivorensis A. Chev, é árvore de origem africana, especificamente da porção ocidental do continente, distribuída da costa leste de Costa do Marfim e Camarões ao sul da Angola (Verzignassi et al., 2009), podendo ocorrer no Congo (Lemmens, 2008; Orwa et al., 2009). A espécie também é empregada em plantios florestais em sua área de ocorrência, além de ser plantada na Ásia tropical, América do Sul e Austrália (FAO, 1997; Pinheiro et al., 2011; Dickson et al., 2011). Possui diversas denominações vernaculares, tais como: African mahogany e Nigerian mahogany, pelos ingleses; Acajou D’Afrique, pelos franceses e belgas; Afrikaans mahoganie, na Holanda, Khaya mahagoni, na Alemanha e mogno africano em Portugal e no Brasil (Falesi \& Baena, 1999).

A Khaya ivorensis é planta heliófila, porém tolerante a sombra durante a fase jovem (Foli, 2000; Batista, 2010), sendo ainda classificada como espécie pioneira ou secundária tardia e emergente enquanto posição sociológica (Budowski, 1965; Denslow, 1987), regenerando em clareiras abertas na floresta (Swaine \& Whitmore, 1988). É classificada ainda, contraditoriamente aos supracitados, como uma espécie não pioneira, demandadora de luz (Hawthorne, 1990 apud Tchoundjeu \& Leakey, 1996).

Em condições naturais, segundo Pinheiro et al. (2011), a K. ivorensis pode atingir diâmetro a 1,30 metros do solo (DAP) máximo de 2,1 metros e altura de até 60 metros. O fuste dessa árvore é reto, cilíndrico e sem galhos até uma altura de 30 metros.

Além das propriedades ótimas da madeira da $K$. ivorensis, a espécie em seus locais de origem apresenta diversos usos medicinais (Zhang et al., 2009; Tepongning et al., 2011) tais como no tratamento de malária (Tepongning et al., 2013), além dos diversos usos não madeireiros (Taiwo \& Ogunbodede, 1995; Pinheiro et al., 2011).

Silva (2010) e Carvalho et al. (2010) quantificaram a densidade básica da madeira da espécie em $471 \mathrm{~kg} . \mathrm{m}^{-3}$, qualificando-a como média. $\mathrm{O}$ valor foi baixo provavelmente devido à idade jovem das árvores avaliadas - 10 anos -, provindas do município de Seropédica, RJ, com $30 \mathrm{~cm}$ de DAP e $8 \mathrm{~m}$ de altura de fuste.

No Brasil, a espécie foi introduzida inicialmente no norte do país por meio de sementes doadas ao pesquisador Ítalo Falesi no ano de 1976, porém apenas em 1989 as árvores oriundas do plantio dessas sementes se reproduziram e permitiram a difusão da espécie no país. Assim, devido às restrições impostas ao comércio do mogno brasileiro (Swietenia macrophylla), à semelhança entre as propriedades da madeira das diferentes espécies de mogno e, aliada a alta resistência ao ataque do microlepidóptero Hypsiphyla grandella, principal praga do mogno nativo quando cultivado em plantios puros (Poltronieri et al., 2000; Conde, 2006; Krisnawati et al., 2011), os plantios de mogno africano se expandiram em diferentes regiões do país. 
Pesquisas de diferentes naturezas utilizando a espécie estão se iniciando no Brasil (Falesi \& Baena, 1999; Gasparotto et al., 2001; Poltronieri et al., 2002; Castro et al., 2008; Gomes, 2010; Tremacoldi et al., 2013; França et al., 2016; Corcioli et al., 2016). Siqueira et al. (2002), ao avaliarem o comportamento de plantios florestais utilizando 18 espécies exóticas em Sergipe constataram que o mogno africano possuiu crescimento lento quando comparado às demais espécies, não sobrevivendo às condições da baixada litorânea. Carvalho et al. (2010) verificaram, após testes físico-mecânicos, que a madeira de mogno africano apresentou densidade aparente e básica considerada média, avaliação regular para cavilha, porém característica boa para utilização na indústria moveleira. Albuquerque et al. (2013), avaliando a ecofisiologia de plantas jovens de mogno africano, concluíram que são moderadamente tolerantes ao déficit hídrico, viabilizando plantios comerciais em áreas sujeitas a períodos moderados de estiagem. Silva et al. (2008) relataram o potencial da espécie para uso em sistemas agroflorestais na Amazônia, mesmo sendo pouco difundida entre os produtores rurais e ainda carente de estudos.

\section{PLANTIO E MANEJO FLORESTAL DE KHAYA IVORENSIS}

Os plantios mais velhos de Meliaceae, incluindo o gênero Khaya, foram implantados na Nigéria no ano de 1928, mas medições periódicas não foram realizadas até 1954, dificultando o acompanhamento corrente do crescimento (Nokoe \& Okojie, 1984). Resultados baseados em medições contínuas, utilizando um sistema de amostragem bem definido, ainda são escassos na literatura (Appiah, 2013). Trabalhos como os de Foli (2000), Krishnapillay (2002), Ahmad Zuhaidi et al. (2006), Lemmens (2008) e Heryati et al. (2011) retrataram um pouco da experiência na condução de plantios de K. ivorensis na África e na Malásia. Na Austrália, Nikles et al. (2012) apresentaram resultados sobre plantios florestais, porém para $K$. senegalensis.

No Brasil, segundo a Associação Brasileira de Produtores de Mogno Africano, já são aproximadamente 10 mil hectares plantados com a espécie em diferentes estados. Porém, a maioria dos plantios ainda é jovem, apresentando um crescimento inicial médio no estado de Minas Gerais, nos primeiros 12 meses de vida, de 3,9 cm de DAP e 3,0 m em altura (Lopes et al., 2012).

Castro et al. (2008) relataram que em sistemas silvipastoris no Pará o mogno africano pode alcançar altura de fuste de 12 metros e DAP de 22 centímetros aos 7 anos de idade. Falesi \& Galeão (2002) apud Silva (2010), estudando um reflorestamento no Pará, encontraram valores de crescimento para o mogno africano com média de altura total de 8,5 metros e de DAP de 15,5 centímetros aos 5 anos e 8 meses; já aos 6 anos e 4 meses de idade, a média de altura total foi de 9,2 metros e DAP de 17,3 centímetros. Plantios em Minas Gerais têm sido mais produtivos, atingindo valores de altura média de $3 \mathrm{~m}$.ano ${ }^{-1}$ e DAP médio de $4 \mathrm{~cm}$ ano ${ }^{-1}$, próximo aos 5 anos de idade (Figura 1).

Ofori et al. (2007) afirmaram que o comércio contínuo da madeira nativa do mogno africano se encontra ameaçado pela intensa exploração das florestas e pelas dificuldades no estabelecimento de plantações de mogno em seu local de origem, devido ao ataque da broca de ponteira Hypsipyla robusta Moore, mesmo problema ocorre para a $S$. macrophylla em relação à H. grandella. $\mathrm{O}$ ataque da Hypsipyla destrói os brotos terminais, fazendo com que se bifurquem, retardando o crescimento e reduzindo o valor econômico da madeira (Grogan et al., 2002; Lim, 2007; Opuni-Frimpong et al., 2008a). Ofori et al. (2007) aludiram que plantações de K. ivorensis e K. anthotheca, em associação com Azadirachta indica e Albizia lebbeck, deram resposta positiva no controle da praga $H$. robusta.

Hawkes (1976) já alertava que o emprego de plantios florestais poderia ser uma alternativa para reduzir a pressão nas florestas naturais e, ao mesmo tempo, garantir um controle de produção mais uniforme. Porém o autor apontou para o grave risco em se ter uma base genética reduzida e consequente fragilidade ao ataque de pragas e doenças. No Brasil, estudos sobre a variabilidade genética estão se iniciando, uma vez que grande parte das sementes utilizadas nos plantios brasileiros provêm das mesmas matrizes instaladas no estado do Pará.

Jeyanny et al. (2009), ao estudarem o efeito da deficiência de macronutrientes no crescimento e vigor de mudas de Khaya ivorensis concluíram que a falta de N, P, K, Ca e Mg nas mudas manifesta um efeito visual dos sintomas de deficiência nutricional, além de interferir no crescimento e na concentração 

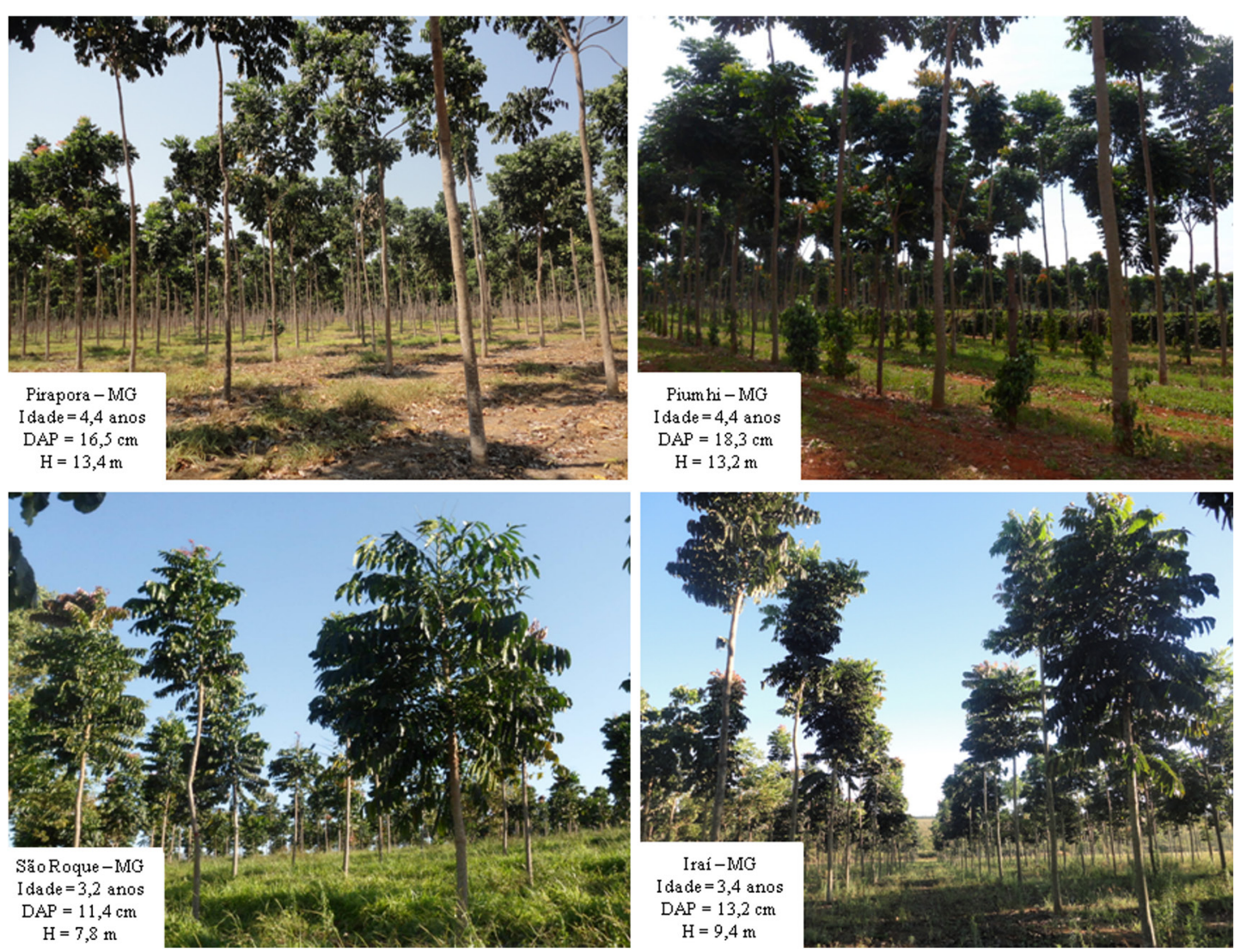

Figura 1. Valores médios de características dendrométricas em plantios de Khaya ivorensis localizados no estado de Minas Gerais.

Figure 1. Mean values of dendrometric variables for Khaya ivorensis plantations located in Minas Gerais state.

de nutrientes nos tecidos das plântulas. Destacou-se a importância do magnésio para o crescimento da muda e do potássio, que afeta diretamente o conteúdo de nutrientes nos tecidos da plântula.

Lemmens (2008), no seu estudo sobre essa espécie, levantou várias tendências de crescimento em condições de plantio. $\mathrm{O}$ autor conclui que o mogno africano pode ser considerado espécie de crescimento médio, exigente de luz e com propriedades de desrama natural (galhos mortos se desprendem do fuste sozinhos). Em solos férteis na Costa do Marfim, plantios com 31 anos de idade e densidade de 70 árvores por hectare atingiram valores médio de produtividade de $8 \mathrm{~m}^{3} \cdot \mathrm{ha}^{-1}$. $\mathrm{ano}^{-1}$, com DAP médio de $57 \mathrm{~cm}$ e altura média de 38,5 metros.

Em plantios na Malásia, em que a rotação esperada é de 30 a 60 anos, produtividade de $7,5 \mathrm{~m}^{3} \cdot \mathrm{ha}^{-1} \cdot$ ano $^{-1}$ foi relatada aos 27 anos de idade, na qual as características médias de crescimento das árvores individuais foram de 1,8 cm.ano ${ }^{-1}$ em DAP e 1 m.ano ${ }^{-1}$ em altura. No mesmo país, aos 40 anos de idade, foram relatadas árvores médias com 23,5 m de altura e 29,5 cm de DAP, e as árvores dominantes com $30 \mathrm{~m} \mathrm{e} 47 \mathrm{~cm}$, respectivamente (Lemmens, 2008).

Dupuy \& Koua (1993) realizaram vários estudos aplicando diferentes técnicas de manejo do mogno africano em plantios na Costa do Marfim. Segundo esses autores, os melhores resultados são obtidos em plantios de alta densidade (espaçamento de $3 \times 3 \mathrm{~m}$ ) empregando desbastes subsequentes ao longo dos anos do plantio, podendo as árvores atingirem altura de 20 metros, aos 20 anos, e DAP de $50 \mathrm{~cm}$ próximo aos 40 anos de idade.

Aminah et al. (2005) relataram sobre o crescimento de um experimento de K. ivorensis na Malásia, utilizando espaçamento de $3 \times 3 \mathrm{~m}$ (1.111 árvores.ha ${ }^{-1}$ ), sendo as mudas plantadas provenientes de estaquia de plantas adultas. Aos 7 anos, o plantio apresentava sobrevivência de $87,8 \%$, com DAP médio de $18,8 \mathrm{~cm}$ (incremento 


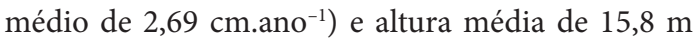
(incremento médio de 2,26 m.ano ${ }^{-1}$ ). Heryati et al. (2011), pesquisando a performance do crescimento e acumulação de biomassa em plantios de K. ivorensis de 5 anos de idade em diferentes classes de solo, também na Malásia, encontraram valores de incremento em volume variando de $43 \mathrm{~m}^{3}$.ha ${ }^{-1}$ a $53 \mathrm{~m}^{3}$.ha $\mathrm{h}^{-1}$, com DAP médio variando de $11,6 \mathrm{~cm}$ a $14,4 \mathrm{~cm}$ e altura média variando de $7,8 \mathrm{~m}$ a $10,6 \mathrm{~m}$.

Quanto ao crescimento da espécie em idades mais avançadas, Ahmad Zuhaidi et al. (1999) relataram o crescimento de um plantio estabelecido em 1957 utilizando espaçamento de $3 \times 3$ metros, localizado na Malásia, em uma área com precipitação anual de 2.000 a $2.900 \mathrm{~mm}$. Os autores apresentaram os valores para o plantio de 40 anos de idade: DAP médio de $29,5 \mathrm{~cm} ; 23,5 \mathrm{~m}$ de altura média; de área basal média de $26,6 \mathrm{~m}^{2}$.ha $\mathrm{a}^{-1}$ e volume médio de $305,5 \mathrm{~m}^{3} \cdot \mathrm{ha}^{-1}$. Já no Brasil, os indivíduos de idade mais avançada, plantados na Embrapa Amazônia Oriental, Belém (Figura 2), são as 4 árvores matrizes das sementes que deram origem à maioria dos plantios no país. Essas árvores possuem, atualmente, 37 anos de idade e foram plantadas isoladas, a aproximadamente 20 metros de distância uma da outra. Enquanto uma dessas árvores apresenta tronco muito tortuoso, as três restantes têm forma excepcional. Atualmente, as três árvores de boa forma possuem DAP médio de $1,3 \mathrm{~m}$, altura total média de $38 \mathrm{~m}$ e altura de fuste de $12,4 \mathrm{~m}$. O elevado crescimento desses indivíduos $\left(3,6 \mathrm{~cm}\right.$. ano $^{-1}$ para DAP e $1 \mathrm{~m}$. ano $^{-1}$ para altura total), em idade tão avançada

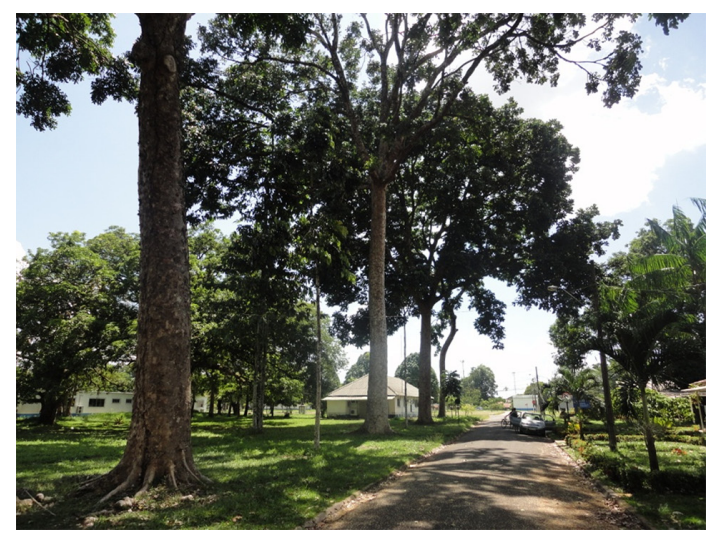

Figura 2. Árvores pioneiras de mogno africano plantadas no Brasil (Belém, PA).

Figure 2. First African mahogany trees planted in Brazil (Belém, PA). mostra o potencial de crescimento extraordinário da espécie. No entanto, é importante ressaltar que para que plantios de mogno africano atinjam porte tão elevado a densidade final deve ser baixa (aproximadamente $17 \mathrm{~N} /$ ha, considerando espaçamento final de $30 \times 20 \mathrm{~m}$ ), obtida por meio de desbastes.

Os plantios de mogno africano instalados na Ásia geralmente contam com um espaçamento inicial de $3 \times 3 \mathrm{~m}$, sendo realizados subsequentes desbastes para reduzir a densidade inicial de 1.111 árvores por hectare para valores em torno de 200 a 350 árvores, almejando um corte final entre 20 e 25 anos. Na África, é comum o emprego do enriquecimento nas florestas naturais, ou seja, a introdução de espécies de interesse comercial na floresta com aplicação de diferentes atividades silviculturais, encontrando-se a cada 3 hectares uma árvore explorável de interesse comercial. Existem, também, plantios nos locais de origem, que são realizados com espaçamentos diversos (Foli et al., 2003), porém, menos preferidos devido ao ataque da broca de ponteiro. No Brasil, até o presente, não existem muitos dados publicados sobre as tendências de crescimento da espécie em questão. Os plantios realizados aqui possuem características distintas, marcadamente o espaçamento mais amplo $(4 \times 6 \mathrm{~m}, 5 \times 5 \mathrm{~m}, 6 \times 6 \mathrm{~m}, 5 \times 8 \mathrm{~m}$, entre outros $) \mathrm{e}$ tratos silviculturais adequados.

\section{COMERCIALIZAÇÃO DA MADEIRA DE KHAYA SPP.}

A madeira do gênero Khaya é amplamente comercializada desde o início do século passado e, devido à exploração predatória, indivíduos com grandes dimensões são raros em alguns de seus locais de origem (Arnold, 2004). O comércio do mogno africano é consolidado devido às características tecnológicas e à beleza de sua madeira, utilizada na indústria moveleira, na construção naval e em sofisticadas construções de interiores (Agbedahunsi et al., 2004; Ward et al., 2008; Opuni-Frimpong et al., 2008c).

Khaya ivorensis possui madeira de coloração vermelha a marrom pálido, densidade básica média de $0,47 \mathrm{~g}$ a $0,58 \mathrm{~g} . \mathrm{cm}^{-3}$ e boa trabalhabilidade (CABI, 2013). Assim, a madeira do gênero Khaya é muito valorizada no mercado internacional: o valor do metro cúbico de toras nativas chega a aproximadamente US\$ 1 mil, conforme registros de importação e 
exportação de diferentes produtos de madeira do gênero Khaya publicados pela International Tropical Timber Organization - ITTO (Tabela 1).

Dados sobre a comercialização de madeira de Khaya ivorensis provinda de florestas nativas localizadas na República do Gana foram relatados pela ITTO $(2008,2009,2010,2011)$ e são apresentados na Tabela 2.

Conforme citado anteriormente, os preços pagos no mercado internacional são referentes a madeira proveniente de florestas tropicais naturais. Assim, preços de mercado da madeira oriunda de plantios ainda são especulados. Segundo ITTO (2012), o Panamá exportou madeira serrada de Khaya spp. pelo valor médio de US $\$ 267 / \mathrm{m}^{3}$. Portanto, esse valor é, até o momento, o único publicado para o comércio de Khaya proveniente de plantios comerciais fora dos locais de origem. Em relatórios mais atuais da ITTO (2016), valores de exportação proveniente em

Tabela 1. Valores monetários por metro cúbico e quantidade de diferentes produtos de Khaya spp. importados e exportados por diferentes países, adaptado de ITTO $(2008,2009,2010,2011)$.

Table 1. Monetary values per cubic meter and quantity of different products of Khaya spp. imported and exported by different countries, adapted from ITTO $(2008,2009,2010,2011)$.

\begin{tabular}{|c|c|c|c|c|c|c|}
\hline \multirow[b]{2}{*}{ País } & \multirow[b]{2}{*}{ Produto $^{\star}$} & \multirow[b]{2}{*}{ Ano } & \multicolumn{2}{|c|}{ Importação } & \multicolumn{2}{|c|}{ Exportação } \\
\hline & & & $\begin{array}{c}\text { Volume } \\
\left(1.000 \mathrm{~m}^{3}\right)\end{array}$ & $\begin{array}{c}\text { Preço } \\
\left(\mathrm{US} \$ \mathbf{m}^{3}\right)\end{array}$ & $\begin{array}{l}\text { Volume } \\
\left(1.000 \mathrm{~m}^{3}\right)\end{array}$ & $\begin{array}{c}\text { Preço } \\
\left(\mathrm{US} \$ \mathbf{m}^{3}\right)\end{array}$ \\
\hline \multirow{13}{*}{ França } & \multirow{5}{*}{ Toras } & 2006 & 22 & 386 & 1 & 778 \\
\hline & & 2007 & 28 & 405 & 1 & 823 \\
\hline & & 2008 & 21 & 456 & 1 & 925 \\
\hline & & 2009 & 14 & 365 & $<1$ & 741 \\
\hline & & 2010 & 16 & 403 & $<1$ & 819 \\
\hline & \multirow{5}{*}{$\begin{array}{l}\text { Madeira } \\
\text { laminada }\end{array}$} & 2006 & 12 & 827 & $<1$ & 3924 \\
\hline & & 2007 & 11 & 1178 & $<1$ & 2307 \\
\hline & & 2008 & 10 & 1273 & $<1$ & 4255 \\
\hline & & 2009 & 4 & 1096 & $<1$ & 3641 \\
\hline & & 2010 & 9 & 1012 & $<1$ & 3475 \\
\hline & \multirow{3}{*}{$\begin{array}{l}\text { Painel de } \\
\text { madeira }\end{array}$} & 2006 & 2 & 775 & 11 & 1284 \\
\hline & & 2007 & 3 & 748 & 10 & 1558 \\
\hline & & 2008 & - & - & 9 & 1634 \\
\hline \multirow{12}{*}{ Portugal } & \multirow{4}{*}{ Toras } & 2006 & 18 & 488 & 1 & 664 \\
\hline & & 2007 & 16 & 481 & 1 & 684 \\
\hline & & 2008 & 9 & 573 & $<1$ & 1695 \\
\hline & & 2009 & 6 & 1428 & $<1$ & 1397 \\
\hline & \multirow{4}{*}{$\begin{array}{l}\text { Madeira } \\
\text { laminada }\end{array}$} & 2006 & $<1$ & 1372 & - & - \\
\hline & & 2007 & $<1$ & 1107 & - & - \\
\hline & & 2008 & 1 & 460 & 1 & 386 \\
\hline & & 2009 & $<1$ & 303 & $<1$ & 663 \\
\hline & \multirow{4}{*}{$\begin{array}{c}\text { Painel de } \\
\text { madeira }\end{array}$} & 2006 & 3 & 808 & - & - \\
\hline & & 2007 & 1 & 981 & - & - \\
\hline & & 2008 & $<1$ & 1583 & - & - \\
\hline & & 2009 & $<1$ & 875 & - & - \\
\hline \multirow{4}{*}{ Coréia do Sul } & \multirow{2}{*}{ Toras } & 2008 & $<1$ & 787 & - & - \\
\hline & & 2009 & $<1$ & 787 & $<1$ & 269 \\
\hline & \multirow{2}{*}{$\begin{array}{c}\text { Madeira } \\
\text { laminada }\end{array}$} & 2008 & $<1$ & 2667 & - & - \\
\hline & & 2009 & $<1$ & 2030 & $<1$ & 1217 \\
\hline \multirow{2}{*}{ Holanda } & \multirow{2}{*}{ Toras } & 2009 & $<1$ & 840 & - & - \\
\hline & & 2010 & $<1$ & 742 & - & - \\
\hline \multirow{2}{*}{ Austrália } & \multirow{2}{*}{$\begin{array}{l}\text { Madeira } \\
\text { serrada }\end{array}$} & 2009 & 1 & 1283 & - & - \\
\hline & & 2010 & 1 & 1103 & - & - \\
\hline
\end{tabular}

${ }^{*}$ Os dados de quantidade foram ponderados para os casos que relataram mais de uma espécie no mesmo produto comercializado. 
Tabela 2. Valores monetários por metro cúbico e quantidade de diferentes produtos de Khaya ivorensis exportados da República do Gana (ITTO 2008, 2009, 2010, 2011).

Table 2. Monetary values per cubic meter and quantity of different products of Khaya ivorensis exported from the Republic of Ghana (ITTO 2008, 2009, 2010, 2011).

\begin{tabular}{|c|c|c|c|}
\hline Produto & Ano & $\begin{array}{c}\text { Volume } \\
\left(1.000 \mathrm{~m}^{3}\right)\end{array}$ & $\begin{array}{c}\text { Preço } \\
\left(\mathrm{US} \$ / \mathbf{m}^{3}\right)\end{array}$ \\
\hline \multirow{5}{*}{$\begin{array}{c}\text { Madeira } \\
\text { serrada }\end{array}$} & 2006 & 17 & 755 \\
\hline & 2007 & 15 & 878 \\
\hline & 2008 & 13 & 884 \\
\hline & 2009 & 5 & 545 \\
\hline & 2010 & 8 & 844 \\
\hline \multirow{5}{*}{$\begin{array}{l}\text { Madeira } \\
\text { laminada }\end{array}$} & 2006 & 5 & 1938 \\
\hline & 2007 & 4 & 2341 \\
\hline & 2008 & 4 & 1799 \\
\hline & 2009 & 2 & 1559 \\
\hline & 2010 & 2 & 1507 \\
\hline \multirow{5}{*}{$\begin{array}{l}\text { Painel de } \\
\text { madeira }\end{array}$} & 2006 & 7 & 449 \\
\hline & 2007 & 10 & 437 \\
\hline & 2008 & 10 & 480 \\
\hline & 2009 & 11 & 552 \\
\hline & 2010 & 10 & 516 \\
\hline
\end{tabular}

Gana da madeira nativa de K. ivorensis seca em estufa atingiram 993 euros $/ \mathrm{m}^{3}$ e da madeira nativa seca ao ar livre, 848 euros $/ \mathrm{m}^{3}$.

Sabe-se da importância das plantações florestais para garantia do fornecimento interno e externo de madeira (Hartley, 2002). A área mundial de plantio é crescente, estudos recentes argumentam que antes do ano de 2050 todos os produtos madeireiros serão derivados de plantios florestais manejados (Dyck, 2003).

\section{CONSIDERAÇÕES FINAIS}

Plantios florestais manejados de forma sustentável possuem múltiplas funções ambientais que são importantes tanto em escala nacional como internacional, além de desempenhar um papel vital no desenvolvimento sustentável, suprindo as exigências do mercado consumidor.

O Brasil é referência em termos de silvicultura e velocidade de crescimento de espécies arbóreas, fato atestado pelo crescimento satisfatório de plantios jovens de mogno africano localizados principalmente em Minas Gerais, bem como pelo porte e desenvolvimento de árvores de mogno africano mais velhas, plantadas no Pará. Porém estudos aprofundados quanto à viabilidade da implantação da Khaya ivorensis no país devem ser desenvolvidos, pesquisas de outra natureza que permitam o manejo adequado da espécie, a fim de garantir o retorno econômico desejável e a correta domesticação.

\section{AGRADECIMENTOS}

Os autores expressam sinceros agradecimentos aos proprietários de plantios de mogno africano, destacando: Ricardo Tavares, Carlos Rebelatto, Marcos Soares Rezende, em especial, à atenção dada pelo Eng. Agrônomo João Emílio D. Matias e pela Associação Brasileira de Produtores de Mogno Africano.

\section{STATUS DA SUBMISSÃO}

Recebido: 3 fev., 2014

Aceito: 21 abr., 2016

\section{AUTOR(ES) PARA CORRESPONDÊNCIA}

\section{Andressa Ribeiro}

Departamento de Engenharias, Universidade Federal do Piauí - UFPI, Campus Profa . Cinobelina Elvas, Rodovia Municipal Bom Jesus-Viana, Km 01, Planalto Horizonte, CEP 64900-000, Bom Jesus, PI, Brasil e-mail: andressa.florestal@ufpi.edu.br

\section{REFERÊNCIAS}

Agbedahunsi JM, Fakoya FA, Adesanya SA. Studies on the anti-inflammatory and toxic effects of the stem bark of Khaya ivorensis (Meliaceae) on rats. Phytomedicine 2004; 11(6): 504-508. PMid:15500261. http://dx.doi. org/10.1016/j.phymed.2003.07.009.

Ahmad Zuhaidi Y, Abdul Rasip AG, Rosdi K, Zainal T, Mohd Parid M. Establishment and management of Khaya Ivorensis plantations. Malasya: Technical Information Handbook; 2006.

Ahmad Zuhaidi Y, Mahat MN, Ghani ARA. Growth of plantation grown Khaya ivorensis in Peninsular Malaysia. Journal of Tropical Forestry 1999; 11: 849-851.

Albuquerque MPF, Moraes FKC, Santos RIN, Castro GLS, Ramos EMLS, Pinheiro HA. Ecofisiologia de plantas jovens de mogno africano submetidas a deficit hídrico e 
reidratação. Pesquisa Agropecuaria Brasileira 2013; 48(1): 9-16. http://dx.doi.org/10.1590/S0100-204X2013000100002.

Aminah H, Intan ZB, Rosdi K, Rozihawati Z, Ahmad Fauzi MS, Hamzah M. Growth performance of some dipterocarps and non-dipterocarps planted from rooted cuttings. In: Proceedings of the 8th Round-Table Conference on Dipterocarps; 2005; Ho Chi Min City. Vietnam; 2005 [citado em 2013 aug 13]. p. 1-6. Disponível em: http:// vafs.gov.vn/en/2006/06/growth-performance-of-somedipterocarps-and-non-dipterocarps-planted-fromrooted-cuttings/

Appiah M. Tree population inventory, diversity and degradation analysis of a tropical dry deciduous forest in Afram Plains, Ghana. Forest Ecology and Management 2013; 295: 145-154. http://dx.doi.org/10.1016/j.foreco.2013.01.023.

Arnold R. Khaya senegalensis: current use from its natural range and its potential in Sri Lanka and elsewhere in Asia. In: Prospects for high-value hardwood timber plantations in the 'dry' tropics of northern Australia [CD-ROM]. Gympie: Private Forestry North Queensland Association Inc; 2004.

Batista F. Mogno Africano, cultivo no estado de Minas Gerais. Piracicaba: Casa do Produtor Rural; 2010.

Budowski G. Distribution of tropical American rainforest species in light of sucessional processes. Turrialba 1965; 15: 40-43.

Carvalho AM, Silva BTB, Latorraca JVF. Avaliação da usinagem e caracterização das propriedades físicas da madeira de mogno africano (Khaya ivorensis A. Chev.). Cerne 2010; 16: 106-114.

Castro AC, Lourenço JB Jr, Santos NFA, Monteiro EMM, Aviz MAB, Garcia AR. Sistema silvipastoril na Amazônia: ferramenta para elevar o desempenho produtivo de búfalos. Ciência Rural 2008; 38(8): 2395-2402. http:// dx.doi.org/10.1590/S0103-84782008000800050.

Centre for Agriculture and Biosciences International CABI. Forestry Compendium [online]. Wallingford: CAB International; 2013 [citado em 2013 aug 13]. Disponível em: www.cabi.org/fc

Conde RAR. Controle silvicultulral e mecânico da broca do mogno Hypsipila grandella (Zeller, 1848) (Lepdoptera; Pyralidae) em sistema agroflorestal [dissertação]. Belém: Universidade Federal Rural da Amazônia; 2006.

Corcioli G, Borges JD, Jesus RP. Deficiência de macro e micronutrientes em mudas maduras de Khaya ivorensis estudadas em viveiro. Cerne 2016; 22(1): 131-128.

D’Afrique A. (Khaya spp). Revue. Bois et Forêts des Tropiques 1979; 183: 33-48.

Denslow JS. Tropical rainforest gaps and tree species diversity. Annual Review of Ecology and Systematics 1987; 18(1): 431-451. http://dx.doi.org/10.1146/annurev. es.18.110187.002243.

Dickson G, Reilly D, Lindsay DAJ. Abstracts from "Darwin 2011: African Mohogany Plantations Industry Forum" [online]. Brisbane: Department of Employment, Economic Development and Innovation; 2011 [citado em 2013 jun. 25]. Disponível em: http://era.deedi.qld.gov.au/2175/4/ Updated_Afican_mahogany_abstracts_Darwin_2011_ final_4102011-sec.pdf

Dupuy B, Koua M. The African mahogany plantations. Their silviculture in the tropical rain forest of the Côted'Ivoire. Bois et Forêts des Tropiques 1993; 236: 25-42.

Dyck B. Benefits of planted forests: social, ecological and economic. In: UNFF Intersessional Experts Meeting on the Role of Planted Forests in Sustainable Forest Management; 2003; Wellington. New Zeland; 2003 [citado em 2013 jun. 25]. Disponível em: http://maxa.maf.govt.nz/mafnet/ unff-planted-forestry-meeting/conference-papers/ benefits-of-planted-forests.htm

Falesi IC, Baena ARC. Mogno Africano (Khaya ivorensis A. Chev.) em sistema silvipastoril com leguminosa e revestimento natural do solo. Belém: Embrapa Amazônia Oriental; 1999. 52 p. (Documentos, 4).

Foli EG, Alder D, Miller HG, Swaine MD. Modelling growth space requirements for some tropical forest tree species. Forest Ecology and Management 2003; 173(1-3): 79-88. http://dx.doi.org/10.1016/S0378-1127(01)00815-5.

Foli EG. Evaluation of the performance of planted native timber species in different ecological zones in Ghana [project PD 1/93. Final Technical Fellowship Report]. Yokohama: International Tropical Timber Organization; 2000. 41 p.

Food Agriculture Organization - FAO. Hardwood plantations in the tropics and subtropics: tropical forest plantation areas 1995 [project: GCP/INT/628/UK]. Rome: FAO; 1997.64 p.

Food Agriculture Organization - FAO. Promotion of valuable hardwood plantations in the tropics: a global overview [report based on the work of F. K Odoom]. Rome: FAO; 2001. Forest Plantation Thematic Papers, Working Paper 4.

França TSFA, França FJN, Arango RA, Woodward BM, Arantes MDC. Natural resistance of plantation grown African mahogany (Khaya ivorensis and Khaya senegalensis) from Brazil to wood-rot fungi and subterranean termites. International Biodeterioration \& Biodegradation 2016; 107: 88-91. http://dx.doi.org/10.1016/j.ibiod.2015.11.009.

Fremlin R. An overview of African mahogany in Africa. In: Abstracts from "Darwin 2011: African Mohogany Plantations Industry Forum"; 2011; Brisbane. Brisbane: Department of Employment, Economic Development and Innovation; 2011. p. 8-9.

Gasparotto L, Hanada RE, Albuquerque FC, Duarte MLR. Mancha areolada causada por Thanatephorus cucumeris em mogno africano. Fitopatologia Brasileira 2001; 26(3): 660-661. http://dx.doi.org/10.1590/S010041582001000300015 . 
Gomes DM. Análise da viabilidade técnica, econômicofinanceiro para implantação da cultura do mogno africano (Khaya ivorensis A. Chev.) na região oeste de Minas Gerais [monografia]. Curitiba: Universidade Federal do Paraná; 2010. $70 \mathrm{f}$

Grogan J, Barreto P, Veríssimo A. Mogno na Amazônia Brasileira: ecologia e perspectivas de MANEJO. Belém: Imazon; 2002.

Hartley MJ. Rationale and methods for conserving biodiversity in plantation forests. Forest Ecology and Management 2002; 155(1-3): 81-95. http://dx.doi. org/10.1016/S0378-1127(01)00549-7.

Hawkes JG. Introduction. In: Tropical trees: variation, breeding and conservation. London: Linnean Society; 1976. Symposium Series, n. 2.

Heryati Y, Belawan D, Abdu A, Mahat MN, Abdul-Hamid $\mathrm{H}$, Majid NM et al. Growth performance and biomass accumulation of a Khaya ivorensis plantation in three soil series of ultisols. American Journal of Agricultural and Biological Sciences 2011; 6(1): 33-44. http://dx.doi. org/10.3844/ajabssp.2011.33.44.

Ibrahim JA, Ayodele EA, Jegede AI, Kunle YF. Comparative studies on Khaya A. Juss (Meliaceae) in Nigeria. African Journal of Biotechnology 2006; 5(11): 1154-1160.

International Tropical Timber Organization - ITTO. Annual review and assessment of the world timber situation [online]. Yokohama: ITTO; 2008 [citado em 2013 set 15]. Disponível em: http://www.itto.int/annual_review/

IUCN. The IUCN Red List of Threatened Species: version 2013.1 [online]. 2013. [citado em 2013 jul 4]. Disponível em: www.iucnredlist.org

Jeyanny V, Ab Rasip AG, Wan Rasidah K, Ahmad Zuhaidi Y. Effects of macronutrient deficiencies on the growth and vigour of Khaya ivorensis seedlings. Journal of Tropical Forest Science 2009; 21(2): 73-80.

Khairul Alam MD, Basak SR, Alam S. Khaya anthotheca (Welw.) C. Dc. (Meliaceae): an exotic species in Bangladesh. Bangladesh Journal of Plant Taxonomy 2012; 19(1): 95-97.

Krishnapillay B. A manual for forest plantation establishment in Malaysia. Malayan Forest Records 2002; 45: 13-23.

Krisnawati H, Kallio M, Kanninen M. Swietenia macrophylla King: ecology, silviculture and productivity. Bogor: CIFOR; 2011. 24 p.

Lamb FB. On further defining mahogany. Economic Botany 1963; 17(3): 217-232. http://dx.doi.org/10.1007/ BF02859439.

Lamb FB. "Mahogany": a name in controversy. American Speech 1967; 42(3): 219-226. http://dx.doi.org/10.2307/453351.

Lamprecht H. Silvicultura nos trópicos: ecossistemas florestais e respectivas espécies arbóreas - possibilidades e métodos de aproveitamento sustentado. Eschborn: Sociedade Alemã de Cooperação Técnica; 1990. 342 p.
Lemmens RHMJ. Khaya ivorensis A.Chev [online]. Wageningen: PROTA; 2008 [citado em 2013 jul 18]. Disponível em: www.prota4u.org/search.asp

Lim GT. Enhancing the weaver ant, Oecophylla smaragdina (Hymenoptera: Formicidae), for biological control of a shoot borer, Hypsipyla robusta (Lepidoptera: Pyralidae), in Malaysian mahogany plantations [tese]. Virginia: Virginia Polytechnic Institute and State University; 2007.

Lopes ED, Uchôas EG, Gomes JM, Collares RA. Desempenho inicial no campo de mogno africano implantado em área de pastagem na região semi-árida do médio Vale do Jequitinhonha. In: Anais II Simpósio de Integração LavouraPecuária-Floresta; 2012; Montes Claros. Montes Claros: Universidade Federal de Minas Gerais; 2012. p. 131-136.

Nikles DG, Reilly DF, Dickinson GR, Lee DJ. African mahogany (Khaya senegalensis) plantations Australia: status, needs and progress. In: Anais Australian Forest Growers Conference; 2012; Gympie. Queensland Government; 2012 [citado em 2013 jul 18]. p. 1-8. Disponível em: http://era.daf.qld.gov.au/3568/1/DG_Nikles_et_al_AFG_ paper_2012_final.pdf

Nokoe S, Okojie JA. Relationship of stand attributes of some plantation Mahoganies with estimated Weibull parameters. Ecological Modelling 1984; 24(3-4): 231-240. http://dx.doi.org/10.1016/0304-3800(84)90043-7.

Ofori DA, Opuni-Frimpong E, Cobbinah JR. Provenance variation in Khaya species for growth and resistance to shoot borer Hypsipyla robusta. Forest Ecology and Management 2007; 242(2-3): 438-443. http://dx.doi. org/10.1016/j.foreco.2007.01.090.

Opuni-Frimpong E, Karnosky DF, Storer AJ, Abeney EA, Cobbinah JR. Relative susceptibility of four species of African mahogany to the shoot borer Hypsipyla robusta (Lepidoptera: Pyralidae) in the moist semideciduous forest of Ghana. Forest Ecology and Management 2008a; 255(2): 313-319. http://dx.doi.org/10.1016/j.foreco.2007.09.077.

Opuni-Frimpong E, Karnosky DF, Storer AJ, Cobbinah JR. Silvicultural systems for plantation mahogany in Africa: Influences of canopy shade on tree growth and pest damage. Forest Ecology and Management 2008b; 255(2): 328-333. http://dx.doi.org/10.1016/j.foreco.2007.09.078.

Opuni-Frimpong E, Karnosky DF, Storer AJ, Cobbinah JR. Key roles of leaves, stockplant age, and auxin concentration in vegetative propagation of two African mahoganies: Khaya anthotheca Welw. and Khaya ivorensis A. Chev. New Forests 2008c; 36(2): 115-123. http://dx.doi.org/10.1007/ s11056-008-9087-6.

Orwa C, Mutua A, Kindt R, Jamnadass R, Simons A. Agroforestree database: a tree reference and selection guide version 4.0 [online]. 2009. [citado em 2013 jul 18]. Disponível em: www.worldagroforestry.org/af/treedb

Pinheiro AL, Couto L, Pinheiro DT, Brunetta JMFC. Ecologia, silvicultura e tecnologia de utilizações dos mognos- 
africanos (Khaya ssp.). Viçosa: Sociedade Brasileira de Agrossilvicultura; 2011.

Poltronieri LS, Albuquerque FC, Trindade DR, Duarte MLR. Identificação de doenças em mogno-africano no Estado do Pará. Belém: Embrapa Amazônia Oriental; 2000. 13 p. Circular Técnica, n. 18.

Poltronieri LS, Albuquerque FC, Trindade DR, Duarte MLR. Identificação e controle da rubelose em mogno-africano no Estado do Pará. Belém: Embrapa Amazônia Oriental; 2002. 2 p. Comunicado Técnico, n. 68 .

Reilly DF, Robertson RM. Evaluation of the Wood Quality and Utilisation Potential of Plantation grown Khaya senegalensis (African Mahogany). Australia: Northern Territory Government. 2006. 93 p.

Revels CS. Concessions, conflict, and the rebirth of the Honduran mahogany trade. Journal of Latin American Geography 2003; 2(1): 1-17. http://dx.doi.org/10.1353/ lag.2004.0014.

Silva BTB. Avaliação da usinagem e caracterização das propriedades físicas da madeira de mogno africano (Khaya ivorensis A. Chev.) [monografia]. Seropédica: Instituto de Florestas, Universidade Federal Rural do Rio de Janeiro; 2010.

Silva PTE, Brienza S Jr, Yared JAG, Barros PLC, Maciel MNM. Principais espécies florestais utilizadas em sistemas agroflorestais na Amazônia. Revista de Ciências Agrárias (Belém) 2008; 49: 127-144.

Siqueira ER, Ribeiro FE, Carvalho PER, Drumond MA. Comportamento inicial de espécies florestais exóticas na Região da Mata Atlântica de Sergipe. Revista Árvore 2002; 26(L): 13-17.

Swaine MD, Whitmore TC. On the definition of ecological species groups in tropical rain forests. Vegetatio 1988; 75(1-2): 81-86. http://dx.doi.org/10.1007/BF00044629.

Taiwo EA, Ogunbodede RA. Production of tannin adhesives from bark of Nigerian trees. Wood Science and Technology 1995; 29(2): 103-108. http://dx.doi.org/10.1007/ BF00229339.

Tchoundjeu Z, Leakey RRB. Vegetative propagation of African mahogany: effect of auxin, node position, leaf area and cutting length. New Forests 1996; 11(2): 125-136. http://dx.doi.org/10.1007/BF00033408.

Tepongning RS, Lucantoni L, Nasuti CC, Dori GU, Yerbanga SR, Lupidi G et al. Potential of a Khaya ivorensis - Alstonia boonei extract combination as antimalarial prophylactic remedy. Journal of Ethnopharmacology 2011; 137(1): 743-751. PMid:21742022. http://dx.doi.org/10.1016/j. jep.2011.06.036.

Tepongning RS, Yerbanga SR, Dori GU, Lucantoni L, Lupidi G, Habluetzel A. In vivo efficacy and toxicity studies on Erythrina senegalensis and Khaya ivorensis used as herbal remedies for malaria prevention in Cameroon. European Journal of Medicinal Plants 2013; 3(3): 454-464. http:// dx.doi.org/10.9734/EJMP/2013/3928.

The International Tropical Timber Organization - ITTO. Annual review and assessment of the world timber situation [online]. Yokohama: ITTO; 2009 [citado em 2013 set 15]. Disponível em: http://www.itto.int/annual_review/

The International Tropical Timber Organization - ITTO. Annual review and assessment of the world timber situation [online]. Yokohama: ITTO; 2010 [citado em 2013 set 15]. Disponível em: http://www.itto.int/annual_review/

The International Tropical Timber Organization - ITTO. Annual review and assessment of the world timber situation [online]. Yokohama: ITTO; 2011 [citado em 2013 set 15]. Disponível em: http://www.itto.int/annual_review/

The International Tropical Timber Organization - ITTO. Annual review and assessment of the world timber situation [online]. Yokohama: ITTO; 2012 [citado em 2013 set 15]. Disponível em: http://www.itto.int/annual_review/

The International Tropical Timber Organization - ITTO. Tropical Timber Market Report 2016 [citado em 2016 oct 20]; 20(17): 1. Disponível em: https://itto-d2.r-cms.jp/ files/user/mis/MIS_1-15_October2016.pdf

Tremacoldi CR, Lunz AM, Coelho IL, Boari AJ. Cancro em mogno africano no estado do Pará. Pesquisa Florestal Brasileira 2013; 33(74): 220-224. http://dx.doi. org/10.4336/2013.pfb.33.74.415.

Verzignassi JR, Poltronieri LS, Benchimol RL. Mancha-alvo em mogno-africano no Brasil. Summa Phytopathologica 2009; 35(1): 70-71. http://dx.doi.org/10.1590/S010054052009000100015 .

Ward S, Boshier D, Grogan J. Special issue: Sustainable management of high-value timber species of the Meliaceae. Forest Ecology and Management 2008; 255(2): 265-364. http://dx.doi.org/10.1016/j.foreco.2007.09.047.

Zhang B, Yang SP, Yin S, Zhang CR, Wu Y, Yue JM. Limonoids from Khaya ivorensis. Phytochemistry 2009; 70(10): 1305-1308. PMid:19666181. http://dx.doi. org/10.1016/j.phytochem.2009.07.016. 